Chapter 1

\title{
Abiotic Stress Adaptation: Protein Folding Stability and Dynamics
}

\author{
Martina Ortbauer \\ Additional information is available at the end of the chapter \\ http://dx.doi.org/10.5772/53129
}

\section{Introduction}

Abiotic stress is best defined as any factor exerted by the environment on the optimal functioning of an organism. Abiotic stresses like heat, cold, freezing, drought salinity, flooding or oxidizing agents usually cause protein dysfunction [1]. Protein folding stability is undoubtedly one of the most challenging problems in organisms undergoing stress conditions. Efficient protein repair systems and general protein stability facilitate survival upon sudden changes in the environment. As sessile organisms plants need to adopt quickly to overcome various environmental stresses during their lifespan. Recently, most emphasis is being directed towards an understanding of how plants recognize external conditions and initiate protective reactions such as mechanisms through which protein function is protected and maintained. Proteins are biological macromolecules involved in virtually every biological process in a living system. The roles played by proteins are varied and complex. Proteins are used for storage and transport of small molecules or ions and control the passage of molecules through the cell membranes essential for metabolic function [2]. Hormones, which transmit information and allow the regulation of complex cellular processes, are important regulators in responses to abiotic stress [3]. Enzymes act as catalysts and increase, with a remarkable specificity, the speed of chemical reactions essential to the organism's survival.

Protein function is dependent on its unique three-dimensional structure that is adopted by the initial folding of the polypeptide chains after translation. Encoded by DNA and synthesized on ribosomes as chains of hundreds of amino acids, each protein must find its characteristic and correct fold, rather than the countless alternatives, in order to function properly [4]. Folding into its native and active structure may involve one or more partially folded intermediate states (Figure 1). It is not surprising that stress induced alterations in the physiological conditions may change the folding process and give rise to protein misfolding and 
aggregation [5]. Folded proteins are generally much less prone to aggregation and degradation but partially unfolded or intrinsically disordered regions of proteins can confer functional advantages, as they allow efficient interaction with binding partners and provide a mechanism for the regulation of cellular processes. Protein dynamics, meaning structural or conformational change with time, are an essential part of regulation of biological activity.
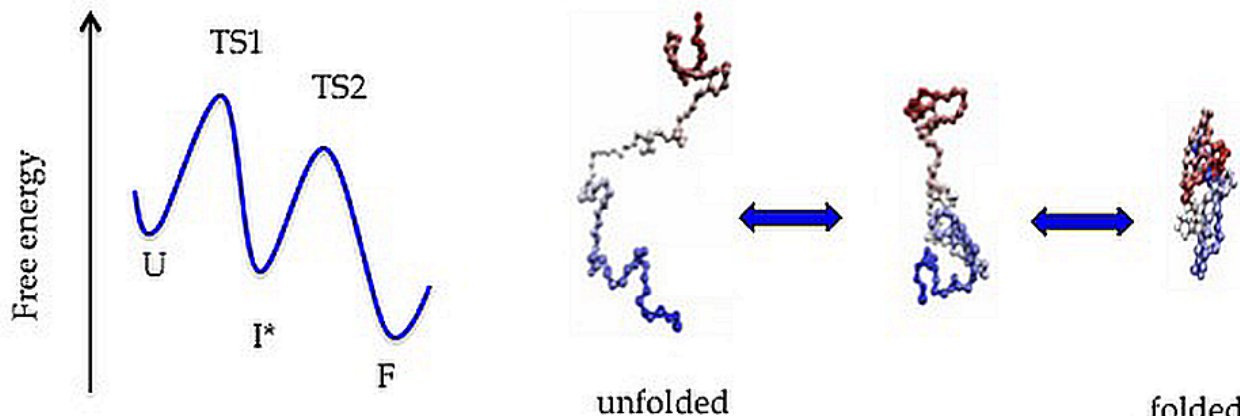

Figure 1. Protein folding involving a partially folded intermediate state. The two transition states (TS1, TS2) are separated by a metastable intermediate $\left(I^{*}\right)$, modified from $[6,7]$. The driving force for protein folding is the search for lower free energy states, separated by free energy barriers. The free energy of a protein in solution is highly dependent on temperature, pressure and solvent conditions

Many cellular processes are coupled to protein folding and unfolding, a process that is highly sensitive to rapid changes in environmental conditions such as denaturant concentration, temperature or $\mathrm{pH}$. In determining the conformational properties of proteins, it is therefore important to include solvent and co-solvent conditions.

Protein conformation and activity can differ markedly between diluted and crowded environments. The diverse and highly specific function of proteins is a consequence of their sophisticated, individual surface pattern regarding shape, charge and hydrophobicity that is a consequence of the three-dimensional structure of polypeptide chains. The stability of proteins results from a number of counteracting enthalpic and entropic contributions. Native states represent the most stable conformation under equilibrium. This does not necessarily mean that protein function is restricted to well-defined folded states. Internal dynamics play an important role in protein function. In vivo folding, catalytic function, transport and degradation of proteins all involve transitions between different conformations. Locally unfolded or disordered regions of a protein allow efficient interaction with binding partners and thus the regulation of cellular mechanisms. Identifying and defining the rules for protein folding and unfolding is fundamental for our understanding how living systems cope with abiotic stresses. Advanced experimental methods continue to be developed to elucidate the sheer complexity of protein folding and unfolding and the mechanisms of preserving functional folds under stress conditions. 


\section{Protein folding and abiotic stress}

A striking feature of protein folding is that the overall mechanism follows simple physical rules, but examination in finer detail reveals a much greater complexity [8]. The protein structure-function paradigm has been reassessed with the discovery of partially unfolded or intrinsically disordered proteins that are fully functional. These proteins are widely distributed in eukaryotes and fulfill crucial biological functions like transcriptional regulation, signal transduction [9], enzyme catalysis and protein ligand interactions. They contain nativelike secondary structure elements but lack the tertiary interactions of folded proteins. One has to keep in mind that protein function is protected by stabilization of well-defined structural regions but is largely dependent on protein motion and dynamics. NMR dynamic experiments indicate that protein conformational exchange spans a variety of time scales ranging from picoseconds to milliseconds [10]. Complete description of protein function, that may involve motion, requires an understanding of the molecular dynamics [11]. Many proteins form partially folded intermediate states along their folding-pathway. To search for correlations between function, structure and dynamics, it is essential to include all states formed at equilibrium $[12,13]$ in order to characterize protein dynamics under unfavorable environmental conditions.

Protein conformations and interconversion between different states are largely modified by internal and external signals such as ligand binding, phosphorylation or cleavage, molecular recognition or environmental changes [14]. In vivo, protein folding occurs spontaneously, meaning that proteins permanently exchange between folded, partially folded, locally unfolded and unfolded states during the period from protein synthesis until their degradation. According to the energy landscape theory, the free energy barriers connecting these states are small [15], suggesting that minor perturbations in vivo can have significant effects on the populations of different states and hence protein function. Intermolecular forces that drive protein folding generally stabilize both folded and unfolded states, but an altered balance in these forces can result in aggregation or misfolding to non-functional proteins [16]. Protein unfolding, misfolding and aggregation are a common threat to the living cell, especially when undergoing abiotic stress. To cope with stress, plants have developed various mechanisms to facilitate protein folding and to suppress protein misfolding.

\section{Stability versus flexibility - How to protect protein function?}

Stabilizing proteins in their functional conformation is one of the great challenges in plant stress metabolism. Stress induced alterations in the structural and energy landscape of proteins affect and may inhibit both protein-ligand and protein-protein interactions. Small molecules typically bind proteins in small cavities, whereas proteins recognize large surface areas [17]. Thus, protein function is a balancing act between structural stability and the conformational flexibility needed for protein function. Protein stability results from stabilizing and destabilizing interactions of the polypeptide chains that slightly favor the folded state as compared to partially folded or unfolded states under physiological conditions (Figure 2). 


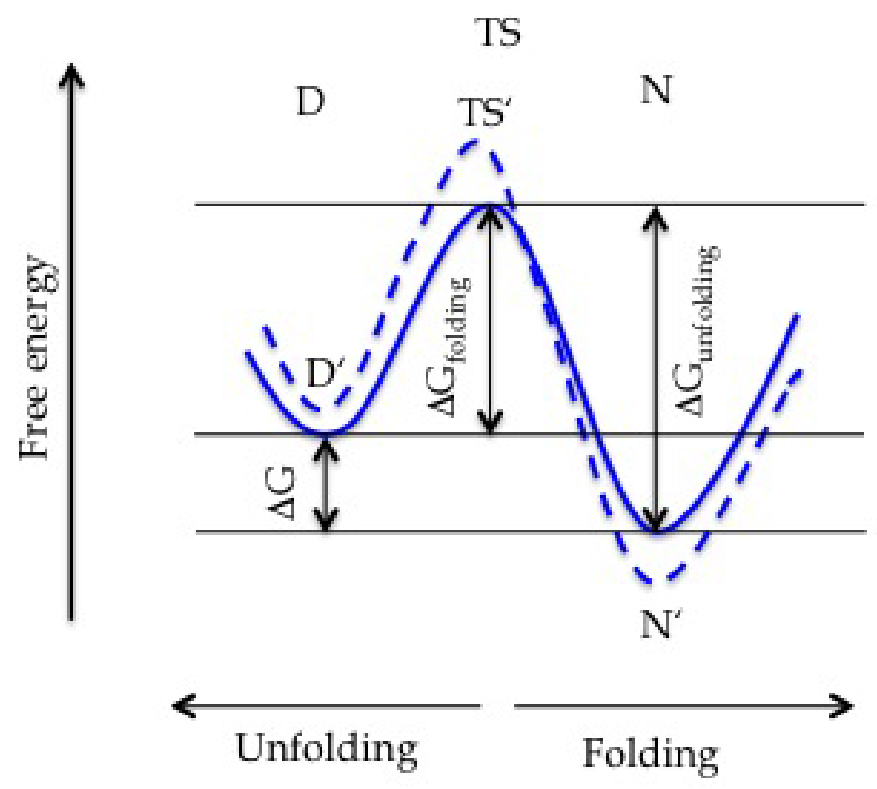

Figure 2. Free energy model for protein folding and unfolding. Stabilization of the native state can be achieved by destabilization of the denatured state $\left(\mathrm{D}^{\prime}\right)$ or a more stabilized native state $\left(\mathrm{N}^{\prime}\right)$. The free energy barrier (TS') may also be affected

The difference in the free energy $(\Delta G)$ of different states as measured from the reversible transition from the native to the denatured state is small [18]. Externally forced conformational changes in the protein structure lead to a substantial decrease in its stability. The denaturation process causes conformational destabilization by exposing hydrophobic residues to the solvent, normally deeply buried in the interior of a folded protein. The burial of non polar surfaces and the hydrophobic force is considered as the main driving force for protein folding and stability [19] as proteins become thermally more stable upon decreasing hydration levels [20].

Evidence from proteins produced by hyperthermophil microorganisms, which are very thermostable and resistant to chemical denaturation, indicates that this resistance comes from lower protein flexibility and higher protein rigidity [21]. Thermostable proteins tend to be very rigid at mesophilic temperatures $\left(10-45^{\circ} \mathrm{C}\right)$, but allow for greater flexibility at high temperatures, which is essential for their function in their thermophilic environment. It is assumed that intrinsic stability due to increased protein rigidity is important for thermal stabilization, since thermal motion decreases rigidity and enhances flexibility.

\subsection{Assisted folding under stress conditions}

Molecular responses to abiotic stress are complex and highly dependent on the level and duration of stress and on the tissue and organ that is affected. Sensing of environmental 
changes and transduction of stress signals triggers activation of molecular response mechanisms [22]. A general response to environmental stress conditions is the onset of stress proteins that facilitate protein folding and protect proteins from misfolding and aggregation. The targets for these so-called chaperons (heat shock proteins HSP, late embryogenesis abundant LEA proteins) are partially unfolded or misfolded proteins with stretches of hydrophobic residues that are normally buried in the interior of the protein fold now exposed to the surface. Since aggregates of misfolded proteins can be very stable and the energy barriers towards the folded state can be of higher energy, chaperons assist the folding process by helping to overcome the energy barriers and to refold proteins from aggregates [23].

Transcription of many genes is up regulated under stress conditions. Among these genes, several code for stress-induced proteins that act to improve water movement through membranes (water channel proteins), detoxification enzymes or enzymes required for osmolyte biosynthesis [24]. Studies on plants reported that one of the initial responses to water deficit is the induction of osmolyte (Figure 3) production. Changes in protein expression levels are required to regulate osmolyte transport and distribution throughout the plant. The accumulation of low-molecular weight osmolytes (compatible solutes) is well known to protect macromolecular structure from stress-induced damage. Increased intracellular osmolyte concentrations on the other hand may affect protein structure and dynamics. Solvent and (co-)solvent conditions and protein solvent accessibility is of particular importance during stress periods because it influences ionic strength, $\mathrm{pH}$ values and affinity to certain molecular groups on the protein surface.

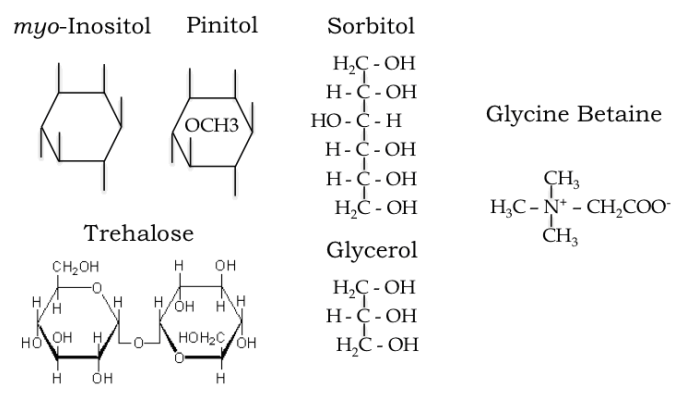

Figure 3. Examples of organic co-solvents (osmolytes): uncharged sugars, polyols and betaines

Accumulated osmolytes within the cells change the interaction of proteins with the solvent [25] by increasing (kosmotropic) or decreasing (chaotropic) the order of water. Kosmotropic, or so-called compensatory co-solvents are well-hydrated molecules with little tendency to aggregate, have no net charge, and strongly hydrogen-bond with water. They are preferentially solubilized within the bulk of water and preferentially excluded from the protein surface, which leads to a decrease in the water diffusion around the protein [26, 27]. Although molecules do not seem to directly interact with the protein surface, they modify protein stability by altering solvent properties. According to the "water structure hypothesis" chaotrop- 
ic co-solvents increase the fraction of more dense species in the hydration water thereby destabilizing protein structure [28]. Molecules that stabilize the surface low-density water and increase the surface tension will stabilize the protein structure (kosmotropes).

Co-solvent effects that alter the water structure are not the sole driving force for increasing or decreasing protein stability. It also has to be considered that the interaction of a co-solvent with the protein surface may not be favorable and thus would destabilize a protein. Due to the fact that unfolded or denatured states comprise a higher solvent and co-solvent accessible surface area, the equilibrium tends to shift to the more compact folded state known as the "excluded volume effect". Among all the interactions that may stabilize or destabilize proteins, a main driving force for protein folding is "hydrophobic interactions". Hydrophobic forces will also be affected in the presence of co-solvents, partly depending on the ability of a solute to be excluded or incorporated in the hydration shell of a certain protein [29].

Increases in temperature, pressure or osmotic stress alter the properties of protein conformation and the hydration state. The free energy change resulting from folding or unfolding depends on the combined effects of the exposure of the interior and non-polar groups and their interaction with water, including changes in water-water interactions.

\subsection{Dynamics in enzymatic activity}

The ability to maintain protein performance under abiotic stress depends on intrinsic stability, chaperon activity, protein turnover and extrinsic stabilization through co-solvents (compatible solutes). Molecular motion as well as protein flexibility and dynamics is highly linked to enzymatic activity, which is clearly dependent on the particular environment of a protein [30].

Hydration status and temperature are the main factors that contribute to the catalytic mechanism. Hydration is necessary for enzyme catalytic function since dry enzymes are less functional, and below a threshold hydration level enzymes are inactive. Protein hydration may also be necessary for diffusion of substrate and product [31]. Temperature is a fundamental environmental stress, as flexibility and functionality of enzymes are highly temperature dependent. Low temperatures result in decreased catalytically activity, which is metabolically not favorable. Increases in the thermal energy will increase enzyme molecules that have the required energy for conformational changes into catalytically active enzymes, showing an increased catalytic rate $\left(k_{\text {cat }}\right)$. High temperatures, on the other hand, can cause the structure to become so loose that substrates and co-factors can no longer bind [32]. Extreme temperatures cause complete denaturation. Osmolyte (glycerol, sorbitol, xylitol, glucose, fructose, saccharose, proline, glycine betaine, myo-Inositol, pinitol, quercitol) protection of enzymes against heat-induced loss of activity has been extensively studied in vitro [33-35]. The particular properties of a protein and the nature of the added osmolyte strongly influence protein thermal stability and enzyme activity. The ability to protect enzymes from heat induced activity loss varies between different osmolytes but preserving enzymes under heat stress seems to be a general feature for these osmolytes. Loss of enzymatic activity under high temperature treatment does occur but is always slower and at higher temperatures when compared to proteins without protective additives. Enzymatic activity tests demonstrate the 
function of osmolytes in preventing heat induced activity loss. To get further insights into folding stability and dynamics of proteins under stress conditions, more detailed analysis and extended methods are needed.

\subsection{Global conformational stability of proteins under stress}

Circular dichroism (CD) spectroscopy has been introduced as a quick and valuable technique for examining the structure and stability of proteins in solution. CD is used for determining whether a protein is folded and for characterizing its secondary structure (alphahelices, beta-strands) and some aspects of the tertiary structure (aromatic amino acids, disulfide bonds). Conformational changes during the acquisition of the native structure are measured in the near-UV (250-350) and far-UV (190-250). This technique has been used widely to determine the folding stability of proteins dependent on temperature, $\mathrm{pH}$ and under denaturant conditions [36,37]. CD is a convenient tool to characterize the interactions between co-solvents and proteins and to find co-solvent conditions that increase the melting temperature or fully refold proteins after thermal unfolding. If the melting is fully reversible, the melting temperature is directly related to conformational stability, and the thermodynamics of protein folding can be extracted from the data [38].

CD studies have been employed to investigate how osmolytes such as glycerol, trehalose and myo-Inositol affect the global folding of native proteins and its thermal unfolding process. CD signals arising from protein chromophors reflect an average of the protein population. The resulting spectrum is a sum of individual spectra arising from secondary structure elements present in the protein sample (Figure 4).

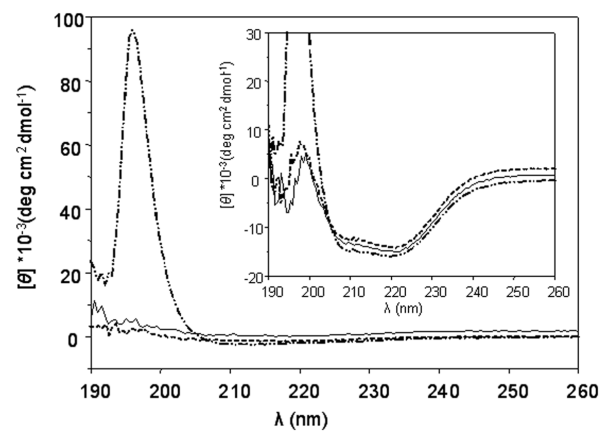

Figure 4. Circular dichroism spectra of Malate Dehydrogenase (insert) in 20mM NaP-buffer at pH 7.0. The far-UV spectrum recorded from 260 to $190 \mathrm{~nm}$ at $20^{\circ} \mathrm{C}$ displays a typical a-helical protein with two negative maxima at 208 and $222 \mathrm{~nm}$. Addition of $0.4 \mathrm{M}$ glycerol (-), myo-Inositol (-) or trehalose (- -) did not change protein secondary structure and did not show self-absorbance in this spectral region [39]

Thermally induced protein unfolding was monitored in the far-UV region by gradually increasing the temperature in the protein sample. Thermal denaturation curves were monitored at a fixed wavelength of 222nm (Figure 5) and acquired data were fitted to a simple thermodynamic unfolding model. The melting temperature, $\mathrm{T}_{\mathrm{m}}$ (midpoint transition temper- 
ature) can be extracted from thermal denaturation curves, reflecting the global stability of the folded versus the unfolded protein. $0.4 \mathrm{M}$ glycerol, myo-Inositol and trehalose increased the melting temperature of malate dehydrogenase by 3 to $5^{\circ} \mathrm{C}$ as compared to proteins alone [39].

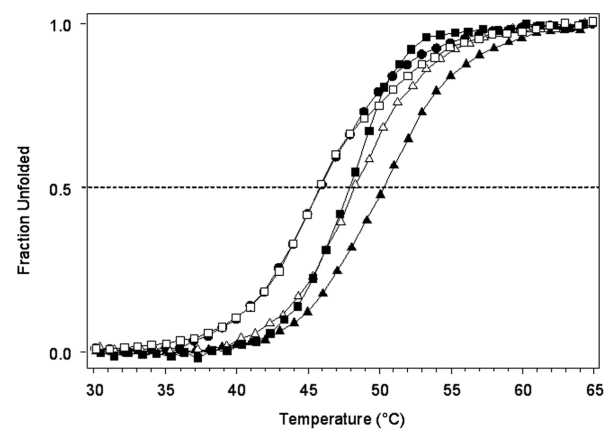

Figure 5. Thermally induced unfolding of Malate Dehydrogenase $(\bullet)$ in the presence of $0.4 \mathrm{M}$ glycerol $(\square)$, trehalose $(\Delta)$, glucose $(\mathbf{-})$ and myo-Inositol $(\mathbf{\Delta})$. The horizontal line indicates the midpoint transition temperature $\left(T_{m}\right)$. Osmolytes and proteins were mixed to protein solution and equilibrated at room temperature prior to heating. Temperature profiles at $222 \mathrm{~nm}$ were recorded for $1{ }^{\circ} \mathrm{C}$ increments in the temperature range $20-90{ }^{\circ} \mathrm{C}$ at a heating rate of $1{ }^{\circ} \mathrm{C} \mathrm{min}{ }^{-1}$ [39]. Thermal unfolding measurements were set up in quartz cuvettes, placed into a Peltier controlled sample holder unit connected to a temperature probe to provide an accurate temperature record. Thermal unfolding curves were analyzed using a sigmoidal curve function according to (Equation 1) [40]:

$$
\theta_{T}=\left[\frac{\left(m_{D} \times T-b_{D}\right)-\left(m_{N} \times T-b_{N)}\right.}{1+\left(T / T_{m}\right)^{m_{T}}}+m_{N} \times T-b_{N}\right]
$$

where $\theta_{\mathrm{T}}$ is the ellipticity at temperature $\mathrm{T}, \mathrm{m}_{\mathrm{T}}$ is the slope of the curve within the transition region, and the inflection point of the curve the melting temperature $\mathrm{T}_{\mathrm{m}}$. At each temperature $b_{N}$ and $b_{D}$ can be extrapolated from the pre- and post-transition baselines, $\left(m_{N} \times T-b_{N}\right)$ and $\left(m_{D} \times T-b_{D}\right)$, respectively. The fraction of unfolded protein can be calculated by subtracting these baselines (Equation 2):

$$
f_{v}=\frac{\theta_{T}-\theta_{N}}{\theta_{v}-\theta_{N}}=\frac{\theta_{T}-\left(m_{N} \times T-b_{N}\right)}{\left(m_{D} \times T-b_{D}\right)-\left(m_{N} \times T-b_{N}\right)}
$$

The stabilization of protein global folds through naturally occurring osmolytes seems to be a general mechanism. Other studies also reported increases in the midpoint transition temperature $\left(\Delta \mathrm{T}_{\mathrm{m}}\right)$ of 2 to $18^{\circ} \mathrm{C}$ upon the addition of $0.1-2 \mathrm{M}$ glycerol, trehalose and sucrose measured on various proteins [41-43]. Additionally, all proteins studied in the presence of osmolytes showed a remarkably retention of secondary structure at $\mathrm{T}_{\mathrm{m}}$ relative to proteins 
alone. Retention of secondary structure in osmolyte solution was monitored even at temperatures where proteins were fully unfolded when heated without additives.

Studies on RnaseA previously showed that increases in $\Delta \mathrm{H}_{\mathrm{m}}$ by the addition of trehalose resulted in a lower $\Delta C p$-vlaue (heat capacity change). [41]. The heat capacity change, $\Delta C p$, is a very sensitive thermodynamic parameter that correlates with the amount of the protein surface that is exposed to the solvent [44]. A decrease in $\Delta \mathrm{Cp}$ upon the addition of osmolytes reflects a lower surface exposed area and/or decreased exposure of hydrophobic groups to the solvent. Decreases in $\Delta \mathrm{Cp}$ may also result in flattening of thermal unfolding curves, leading to conformational stability over a wider range of temperature. This has shown to be an effective strategy for many mesophilic proteins.

The thermal stability of a protein is determined by the response to thermal energy, concerning globally and locally unfolding and the ability to refold into its active conformation. Thermal unfolding was shown to be highly reversible for thermostable proteins of hyperthermophilic organisms. The far-UV CD spectrum of the native protein was identical to that after heat denaturing and re-cooling [45]. Many mesophilic proteins, however, aggregate or precipitate after thermal unfolding making the unfolding process irreversible. Finding co-solvent conditions that facilitate refolding is as important as increasing the melting temperature. Facilitated refolding was observed for ribonuclease that undergoes a reversible denaturation in the presence of trehalose [46].

Taken together, these results from CD measurements reveal that osmolytes stabilize protein global folds under heat by supporting retention of secondary structure elements and aid in refolding of thermally unfolded proteins.

\section{New insights into molecular dynamics of protein folding and unfolding from Nuclear Magnetic Resonance (NMR) spectroscopy}

Internal dynamics of proteins play an important role in their biological function. Proteins do not only exist in well-defined natively folded or fully unfolded states, but also in partially folded intermediate states. The conformational exchange between a folded state and partially folded states is highly sensitive to changes in the environment such as temperature, $\mathrm{pH}$, solvent and co-solvent conditions. In the plant cell, proteins are predestinated to function in environments crowded by macromolecules, metabolites and other co-solvents that facilitate protein folding under non-stress and stress conditions [47]. By measuring protein dynamics, it is therefore important to include (co-)solvent conditions (Figure 6). High-osmolyte accumulation upon stress conditions induces changes in the protein environment. Variable protein folds may be affected slightly different according to their hydrophobic or hydrophilic surface properties, compactness, flexibility, hydrogen bonding patterns, excluded volume effects and the affinity of binding sites for co-solvents or the hydration water. 


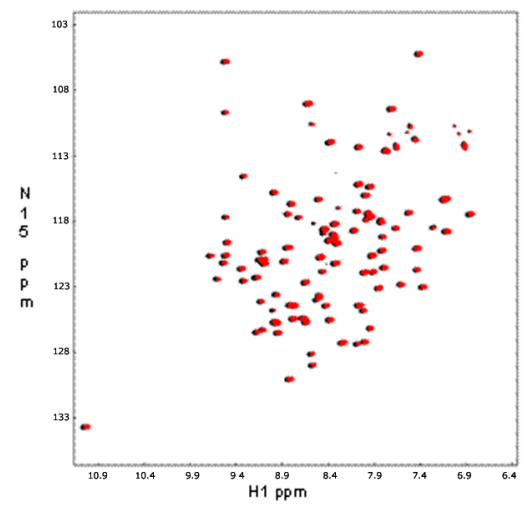

Figure 6. HSQC of the uniformly ${ }^{15} \mathrm{~N}$-labeled KID-binding (KIX) domain of CREB-binding protein (CBP), residues 586-672 (black). Overlaid is a spectrum of KIX in the presence of $0.4 \mathrm{M}$ myo-Inositol (red) showing the intactness of the three-dimensional protein folds upon the addition of an osmolyte. Spectra were acquired on a Varian Inova $800 \mathrm{MHz}$ spectrometer at $26.9^{\circ} \mathrm{C}$

Nuclear Magnetic Resonance (NMR) spectroscopy has greatly contributed to understanding of protein folding by characterizing protein conformation at the level of individual amino acid residues. NMR techniques can be used to monitor temperature dependence of folding and unfolding in order to determine their thermodynamic properties, measure sensitivity to denaturants and address solvent and co-solvent accessibility. NMR experiments provide information at multiple sites within the protein, unlike spectroscopic techniques such as circular dichroism that provide nonspecific information about aromatic side chains and averaged properties of the polypeptide backbone. Heteronuclear NMR relaxation and relaxation dispersion experiments have emerged as powerful tool to study internal dynamics under a wide range of experimental conditions.

\section{NMR relaxation experiments}

Information about protein dynamics, extracted form heteronuclear NMR relaxation studies, is based on measurements of the longitudinal (T1) and transverse (T2) relaxation rate and the heteronuclear NOE, all sensitive for the motion of the N-H bond vector in the protein backbone [11]. Fast atomic motions on a picosecond to nanosecond (ps-ns) time scale are gained from the slower relaxation processes (R1, R2 and NOE) of nuclear spins, measured along the backbone and in the side chains using isotopically labeling $\left({ }^{15} \mathrm{~N}\right)$.

Relaxation data (T1, T2, NOE) can be interpreted according to the "model free" formalism in terms of the internal motional correlation time and an order parameter $\left(\mathrm{S}_{2}\right)$ [48]. In the NMR experiment, order parameters $\left(\mathrm{S}^{2}\right)$ report on the refinement of the $\mathrm{N}-\mathrm{H}$ bond vector. The value of $\mathrm{S}^{2}$ varies from 0 (no motional restriction) to 1 (complete motional restriction) [49]. Backbone segments in highly flexible parts of the protein, not restricted in their motion, have low $\mathrm{S}^{2}$ values, whereas rigid regions show typical high $\mathrm{S}^{2}$ values. Main chain ${ }^{15} \mathrm{~N}$ relaxation data can be analyzed to yield $S^{2}$ order parameters on a per residue basis (Figure 7). 


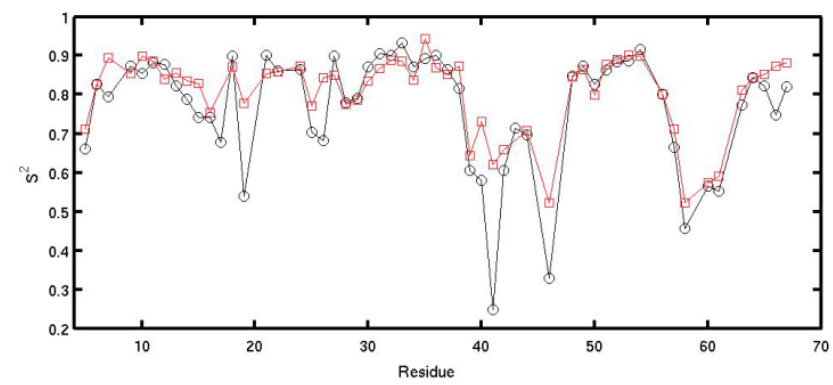

Figure 7. Comparison of $\mathrm{N}-\mathrm{H}$ order parameters $\left(\mathrm{S}^{2}\right)$ of cold shock protein $\mathrm{A}$ (CspA) (red). Addition of $0.4 \mathrm{M}$ myo-Inositol (black) showed an overall increase in protein compactness by rigidification of former flexible parts of the protein $\left(S^{2}=0\right.$ flexible, $S^{2}=1$ rigid)

Order parameter $\left(\mathrm{S}^{2}\right)$ for cold shock protein $\mathrm{A}(\mathrm{CspA})$ showed an overall increase in the presence of the model osmolyte myo-Inositol. Residues in very flexible parts of the protein that have low motional restrictions tend to become more rigid and motional restricted upon the addition of the myo-Inositol. The overall protein compactness increases in the presence of the osmolyte, most profoundly observed in protein regions with high locally structural fluctuations.

\section{(CPMG)-type NMR relaxation dispersion experiments}

NMR relaxation dispersion methods have been introduced enabling studies on protein folding under native conditions without the need for disturbing the equilibrium. Studying protein folding and unfolding requires a thoroughly view of all states including the native state, folding intermediates and the unfolded state [12] as it is increasingly recognized that even small proteins fold via intermediates. Because these intermediates are low populated and short-lived (in the order of ms), their structural characterization has been a difficult task. In NMR relaxation dispersion experiments conformational exchange between a native ground state and low populated partially folded states can be characterized even if states are not visible in NMR spectra [50].

CPMG (Carr-Purcell-Meiboom-Gill)-type NMR relaxation dispersion techniques have been employed to investigate the site-specific conformational exchange processes of proteins on a microsecond-to-millisecond time scale that is highly sensitive to solvent and co-solvent conditions. These experiments are particular useful for simple two state exchange processes, providing information about the kinetics of the exchange process, the relative populations and structural features of invisible states in terms of NMR chemical shifts [51, 52]. Residues that undergo conformational exchange on the $\mu \mathrm{s}-\mathrm{ms}$ time scale contribute to the effective transverse ${ }^{15} \mathrm{~N}$ relaxation rates $\left(\mathrm{R}_{2 . \mathrm{eff}}\right)$. By measuring the increased contribution, $R_{\mathrm{ex}}$, to the effective transverse relaxation rate as a function of CPMG pulse spacing relaxation, typical non-flat dispersion profiles are obtained (Figure 8). 


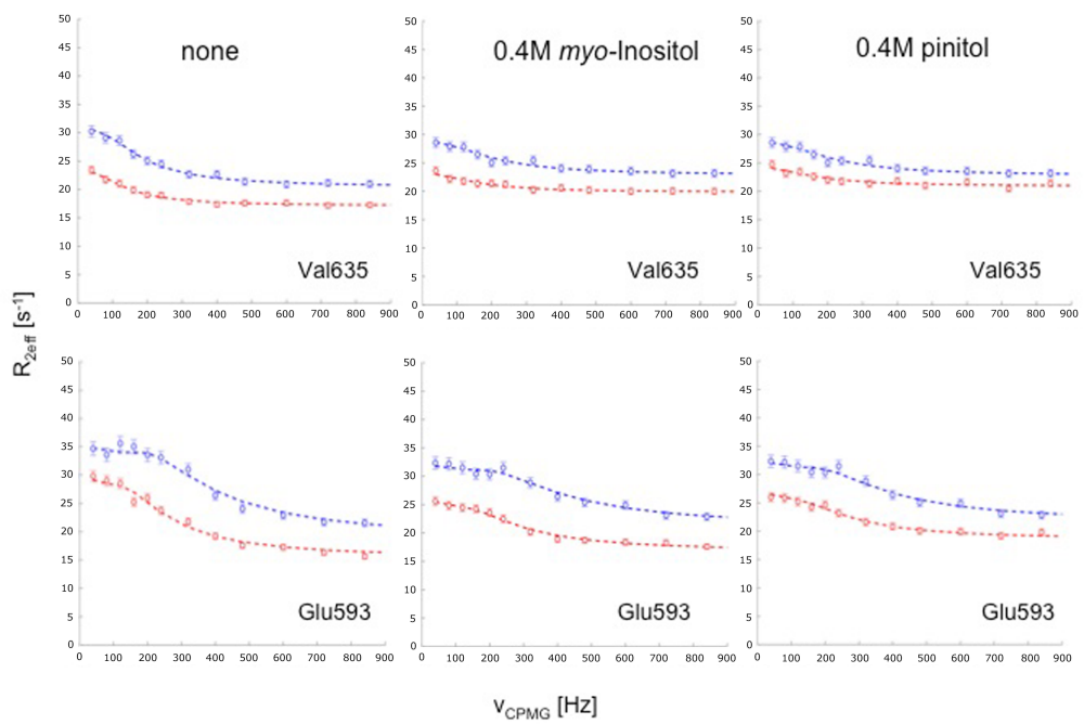

Figure 8. Typical ${ }^{15} \mathrm{~N}$ relaxation dispersion profiles for KIX displaying residues Glu593 and Val635, recorded at static magnetic field strengths of $11.7 \mathrm{~T}$ (red) and $18.8 \mathrm{~T}$ (blue) at $26.9^{\circ} \mathrm{C}$ and in the presence of $0.4 \mathrm{M}$ myo-Inositol and $0.4 \mathrm{M}$ pinitol. Error bars represent uncertainties in relaxation rates. CPMG radio frequency field strengths, $\mathrm{V}_{\mathrm{CPMG}}$, ranged from 40 to $960 \mathrm{~Hz}$, relaxation delays were $50 \mathrm{~ms}$. Spectra were collected as series of two-dimensional data sets. Duplicate data sets were recorded at selected $v_{C P M G}$ values for error analysis. Peak intensities observed from ${ }^{1} \mathrm{H}-{ }^{15} \mathrm{~N}$ spectra were converted into effective relaxation rates $\left(R_{2, \text { eff }}\right)$ and uncertainties in relaxation rates were calculated from repeat experiments. $R_{\text {2.eff }}$ were calculated by numerical modeling of magnetization evolution during the CPMG sequences. Fit curves were obtained by combining the dispersion of all residues in a collective fit to a two-state process

${ }^{15} \mathrm{~N}$ single quantum relaxation dispersion experiments were performed to characterize alterations in the two-site conformational exchange of the KID-binding (KIX) domain of CREBbinding protein (CBP) in the presence of osmolytes under native conditions. Conformational exchange of KIX ${ }^{15} \mathrm{~N}$ backbone resonances has been shown to be in the intermediate to slow time regime. CPMG-type relaxation dispersion data showed that under non-denaturing conditions, KIX permanently exchanges between its folded (native) ground state $(\mathrm{G})$ and a partially unfolded high-energy state (E) that is populated to $3 \pm 0.2 \%$ at $26.9^{\circ} \mathrm{C}$ and $\mathrm{pH} 5.5$. Relaxation dispersion experiments were performed for KIX and in the presence of $0.4 \mathrm{M}$ osmolytes (pinitol, myo-Inositol, quebrachitol, quercitol), operating at static magnetic field strengths of 11.7 and $18.8 \mathrm{~T}$ at $26.9^{\circ} \mathrm{C}$. ${ }^{15} \mathrm{~N}$ relaxation dispersion profiles were fit for each site individually $(\mathrm{G} \leftrightarrow \mathrm{E})$ to yield site-specific values of $\mathrm{G} \rightarrow \mathrm{E}$ and $\mathrm{E} \rightarrow \mathrm{G}$ rate constants $\left(\mathrm{k}_{\mathrm{GE}}\right.$ and $\mathrm{k}_{\mathrm{EG}}$ ) and differences in resonance frequencies between $\mathrm{G}$ and $\mathrm{E}$ states $\left|\Delta \omega_{\text {fit }}\right|$. Dispersion profiles of all sites were then fit to a global two-site model assuming uniform values for $\mathrm{k}_{\mathrm{GE}}$ $\left(\mathrm{k}_{\mathrm{u}}\right)$ and $\mathrm{k}_{\mathrm{EG}}\left(\mathrm{k}_{\mathrm{f}}\right)$, but specific values for $\left|\Delta \omega_{\text {fit }}\right|$ (Table1). Dispersion profiles $\left(\mathrm{R}_{2 . \mathrm{eff}} / \mathrm{V}_{\mathrm{CPMG}}\right)$ are dependent on $k_{f}$ and $k_{u}$ rate constants or the population of the unfolded state $p_{E}$ and the exchange rate constant $\left(k_{e x}=k_{f}+k_{u}\right)$ and on chemical shift differences between the folded and unfolded state $|\Delta \omega|[53]$. 


\begin{tabular}{lccc}
\hline sample & $\mathbf{k}_{\mathrm{f}}\left(\mathbf{s}^{-1}\right)$ & $\mathbf{k}_{\mathrm{u}}\left(\mathbf{s}^{-1}\right)$ & $\mathbf{p}_{\mathrm{E}}(\%)$ \\
\hline none & 574,4 & 16,7 & 2,8 \\
\hline myo-Inositol & 871,5 & 12,3 & 1,4 \\
\hline pinitol & 935,9 & 12,7 & 1,3 \\
\hline quebrachitol & 653,8 & 10,8 & 1,6 \\
\hline quercitol & 661,8 & 13,0 & 1,9 \\
\hline
\end{tabular}

Table 1. Two-site conformational exchange parameters of KIX. The response of $R_{2 \text {.eff }}$ to $v_{\text {CPMG }}$ can be fitted to extract exchange parameters. A two-site exchange model $(\mathrm{G} \leftrightarrow \mathrm{E})$ was fit to ${ }^{15} \mathrm{~N}$ relaxation dispersion data, yielding sitespecific values of $G \rightarrow E$ and $E \rightarrow G$ rate constants $\left(k_{u}\right.$ and $\left.k_{f}\right) . k_{f}$ and $k_{u}$ are the first order rate constants for folding and unfolding transitions, calculated from global fits of ${ }^{15} \mathrm{~N}$ backbone relaxation experiments.

The two-site conformational exchange of KIX between its natively folded ground state and a partially unfolded high-energy state, that represents the equilibrium analog of a folding intermediate [54], was shown to be highly sensitive to the addition of osmolytes. NMR data showed that the composition of these two states differed between the protein in buffer alone and the osmolyte containing sample. Addition of $0.4 \mathrm{M}$ pinitol led to a decrease of more than $50 \%$ in the population of the partially unfolded state $\left(\mathrm{p}_{\mathrm{E}}\right)$. Accordingly, the first order rate constant for folding $\left(\mathrm{k}_{\mathrm{f}}\right)$ increased from $574.4 \mathrm{~s}^{-1}$ to $935.9 \mathrm{~s}^{-1}$ in the presence of pinitol, while the rate constant for unfolding $\left(\mathrm{k}_{\mathrm{u}}\right)$ decreased (from $16.7 \mathrm{~s}^{-1}$ to $12.7 \mathrm{~s}^{-1}$ ). These data provide evidence that even under native conditions osmolytes shift the folding equilibrium towards the folded state. NMR relaxation experiments revealed that osmolytes play an important role on the structure of the folding intermediate, which is the main determinant for protein folding and dynamics. Even though intermediate states are extremely short-lived (in the order of ms), osmolytes greatly influence these states. A decrease in the population of the partially folded state is associated with a destabilization of this state relative to the folded state in the osmolyte containing sample. The interaction of the osmolyte with the protein surface is not favorable and therefore osmolytes are preferentially excluded from the protein surface. Osmolytes indirectly act by changing the properties of water surrounding the protein and hence modify protein-solvent interactions by altering the specific arrangement of the hydrophobic and hydrophilic residues. Folded states are relatively favored over (partially) unfolded states due to their compact structure and smaller surface exposed (solvent accessible) area, leading to a net stabilization of the folded state even under native conditions. Accumulation of high amounts of osmolytes does not seem to be useful under non-stress conditions as they influence protein conformation and dynamics, but they confer great advances to enhance protein stability under stress conditions by counteracting the forces driving protein unfolding. Compact folded conformations are generally less prone to unfolding, misfolding and aggregation that lead to loss of protein function. Increased conformational stability through osmolytes on the other hand allows for greater protein flexibility under elevated temperatures, since thermal motion decreases rigidity and enhances flexibility. This mechanism greatly contributes to preserve protein function under stress conditions in plants. 


\section{Biotechnology approaches for improved abiotic stress tolerance in plants}

Abiotic stress is one of the major causes of crop loss worldwide and restricts certain areas from productive agriculture and even less severe stress makes plants more susceptible to diseases and pests. As sessile organisms plants are exposed to various stresses during their lifespan. With increased understanding of the mechanisms of protein stabilization, advances have been made in genetically engineering more tolerant crop plants.

\subsection{Genetically engineering overproduction of osmolytes}

Progress is being made in genetically modifying plants to accumulate high amounts of osmolytes with the aim to enhance stress tolerance in plants. Transgenic plants have successfully been engineered to accumulate metabolites such as proline, mannitol, glycine betaine and trehalose, which resulted in increased tolerance to various stresses [55-57]. In addition to lowering the osmotic potential and assisting in osmotic adjustment, osmolytes act as hydroxyl radical scavengers and protect macromolecular structure. The accumulation of such metabolites in response to various stresses is a widely distributed phenomenon in the plant kingdom. Some important crop plants, however, are non-accumulators. Genetically introducing mannitol, sorbitol, trehalose or myo-Inositol production in tobacco, Arabidopsis and rice, all species that do not synthesize these compounds naturally, produced enhanced tolerance to salt and drought stress [58-60].

Recently, it has been shown that overexpression of rice (Oryza sativa) choline monooxygenase $(\mathrm{OsCMO})$, the first enzyme in glycine betaine biosynthesis, enhances glycine betaine synthesis in transgenic tobacco plants and resulted in elevated tolerance to salt stress [61]. Although rice has been considered as typical non-accumulator of glycine betaine, this study revealed that the rice containing ortholog of CMO was fully functional in tobacco species. Enhanced tolerance toward salinity, heavy metal, oxidative stress and cold stress was also reported for transgenic tobacco plants when overexpressing rice cystathionine $\beta$-synthase [62] or cold regulated protein CbCOR15b transferred form Capsella bursa-pastoris [63]. Numerous reports show that introducing and enhancing abiotic stress tolerance by the transfer of one or more stress responsive genes between species would be an effective strategy to enhance performance of crop plants in less-productive agricultural areas.

Another strategy for osmolyte overproduction and enhaced plant growth relies on site-directed mutagenesis. $\Delta^{1}$-Pyrroline-5-carboxylase synthase (P5CS), which is feedback inhibited by proline, has been mutated by site-directed mutagenesis, resulting in enzymes that were no longer inhibited. Plants expressing the mutated enzyme had twice the proline levels of WT-plants and exhibited increased tolerance to salt stress [64].

\subsection{Protein engineering}

Protein engineering approaches are being developed for the selection of protein mutations that increase protein stability. New stabilization strategies are based on random mutagene- 
sis and high-throughput screening for thermostability-improving mutations, functional screening or comparison of homologous proteins. Some proteins have been successfully stabilized by the introduction of structural elements from thermophilic and hyperthermophilic homologues [65]. However, the mechanisms underlying thermostability are diverse. Much research has been focused on understanding the stabilization of the hydrophobic core and internal structural elements of proteins $[66,67]$. Recent research has also revealed that protein surfaces have a strong influence on stability and, therefore, have to be taken into consideration. Surface residues are generally more flexible and the protein surface structure is less motional restricted than the compact core. Mutations in the protein surface are therefore supposed to largely affect protein stability and can be introduced to enhance protein stability. Much attention is paid to protein surface salt bridges, as it is known that surface salt bridges become more favorable with increasing temperature and hyperthermophilic proteins tend to have more salt bridges than their mesophilic homologues. Emphasis is made to investigate the contribution of surface salt bridges to enhanced protein stability under stress conditions.

Information from the protein biochemistry field will direct us toward an understanding of the rules for protein folding stability and dynamics with the goal to improve protein stability and stress tolerance in plants.

\section{Conclusion}

Abiotic stresses like desiccation, flooding, high salinity or extreme temperatures are common threats to plants and the optimal function of their metabolism. Protein conformation and stability is dramatically affected by sudden changes in the environment, giving rise to protein unfolding, misfolding and aggregation. Finding the rules for protein folding and unfolding that lead to conformational stability is a matter of ongoing research. Folded states represent the most stable forms under native conditions, but partially folded states that allow for efficient interaction with binding partners are of fundamental importance in biological activity. Studying protein stability under stress conditions has to take protein dynamics, meaning conformational changes of proteins with time, into consideration. Advances have been made in methods to study the conformational exchange in proteins and their folding stability under varying experimental conditions. Nuclear magnetic resonance spectroscopy techniques have been introduced to study the interconversion between folded and partially folded intermediate states. These short-lived, partially folded, states are extremely important for biological activity and play a major role in the energy landscape of proteins. NMR relaxation dispersion experiments revealed that such low populated intermediate folding states are strongly affected by solvent and co-solvent conditions. One of the early onsets of the stress response in plants is the accumulation of osmolytes that serve for osmotic adjustment and protect proteins by maintaining water at the protein surface where it is most needed. NMR dynamic measurements revealed that addition of osmolytes (myo-Inositol, pinitol, quebrachitol and quercitol) lead to a decreased population of the partially folded state by shifting the folding equilibrium towards the folded ensembles. Although osmolytes do not 
directly interact with the protein surface, they alter protein surface properties by changing the water structure and hydrophobic interactions, thereby stabilizing the folded states relative to unfolded states. Even under native conditions, osmolytes were shown to favor the compact folded structure over partially folded structures, consequently leading to alterations in the dynamics of these two states. Thermodynamic considerations assume that osmolytes act by raising the chemical potential of the partially unfolded state relative to the folded state, thereby increasing the (positive) Gibbs energy difference $(\Delta G)$ between folded and unfolded assemblies, thus favoring the folded state with the respect to the unfolded state. By stabilizing compact folded states over unfolded structures even under non-stress conditions, osmolyte accumulation exhibits a great potential to counteract the forces that lead to stress induced protein unfolding. High osmolyte accumulation in plants may not be useful under non-stress conditions as they tend to decrease protein globally and locally flexibility and increase protein overall rigidity. Increased rigidity and overall compactness, however, confer great advances under stress conditions. Compact structures are less prone to unfolding, misfolding, aggregation and degradation. Lower structural flexibility under ambient temperatures allows for greater flexibility under elevated temperatures since thermal motion decreases rigidity and enhances flexibility, which is essential for protein function under stress conditions. Osmolyte production seems to be very effective strategy to adopt plants quickly and with a remarkable plasticity to various changes in their environment. High osmolyte accumulation serves to suppress protein unfolding and misfolding, enhances protein folding stability and facilitate the protein refolding process after complete denaturation. These lessions that we learned from plants and new insights from the protein biochemistry field are taken together for genetically engineering of more tolerant crop plants with the ultimate goal to improve yields in less productive agricultural land.

\section{Acknowledgements}

This work was done in collaboration between the Department of Chemical Physiology of Plants and the Department of Biomolecular Structural Chemistry at the University of Vienna. The author would like to thank Marianne Popp, Robert Konrat, Martin Tollinger and Karin Kloiber for supporting this research and the latter three for providing their expertise in Nuclear Magnetic Resonance Spectroscopy. The author is also grateful to Jürgen König for supporting this work.

\section{Author details}

Martina Ortbauer

Address all correspondence to: martina.ortbauer@univie.ac.at

University of Vienna, Vienna, Austria 


\section{References}

[1] Wang W, Vinocur B, Shoseyov O, Altman A. Role of plant heat-shock proteins and molecular chaperones in the abiotic stress response. Trends in Plant Science. 2004;9(5) 244-52.

[2] Gaci O, Balev S. A General Model for Amino Acid Interaction Networks. World Academy of Science, Engineering and Technology. 2008;44 401-405.

[3] Cramer GR, Urano K, Delrot S, Pezzotti M, Shinozaki K. Effects of abiotic stress on plants: a systems biology perspective. BMC Plant Biology 2011;11 163.

[4] Pain RH. Mechanisms of Protein Folding. Frontiers in Molecular Biology. Oxford University Press Inc., New York; 2000.

[5] Sadana A, Vo-Dinh T. Biomedical implications of protein folding and misfolding. Biotechnology and applied biochemistry. 2001;33 7-16.

[6] Kavraki LE. Dimensionality Reduction Methods for Molecular Motion. http:// cnx.org/content/m11461/1.10/. 2007.

[7] Chen P, Long J, Searle MS. Sequential barriers and an obligatory metastable intermediate define the apparent two-state folding pathway of the ubiquitin-like PB1 domain of NBR1. Journal of Molecular Biology. 2008;376(5) 1463-77.

[8] De Sancho D, Doshi U, Munoz V. Protein folding rates and stability: how much is there beyond size? Journal of the American Chemical Society. 2009;131(6) 2074-5.

[9] Kjaergaard M, Teilum K, Poulsen FM. Conformational selection in the molten globule state of the nuclear coactivator binding domain of CBP. Proceedings of the National Academy of Sciences of the United States of America. 2010;107(28) 12535-40.

[10] Naganathan AN, Doshi U, Fung A, Sadqi M, Munoz V. Dynamics, energetics, and structure in protein folding. Biochemistry. 2006;45(28) 8466-75.

[11] Farrow NA, Zhang O, Szabo A, Torchia DA, Kay LE. Spectral density function mapping using 15N relaxation data exclusively. Journal of Biomolecular NMR. 1995;6(2) 153-62.

[12] Zhang X, Xu Y, Zhang J, Wu J, Shi Y. Structural and dynamic characterization of the acid-unfolded state of hUBF HMG box 1 provides clues for the early events in protein folding. Biochemistry. 2005;44(22) 8117-25.

[13] Lefevre JF, Dayie KT, Peng JW, Wagner G. Internal mobility in the partially folded DNA binding and dimerization domains of GAL4: NMR analysis of the N-H spectral density functions. Biochemistry. 1996;35(8) 2674-86.

[14] Ha JH, Loh SN. Protein Conformational Switches: From Nature to Design. Chemistry-A European Journal. 2012;18(26)7984-99. 
[15] Guo M, Xu Y, Gruebele M. Temperature dependence of protein folding kinetics in living cells. Proceedings of the National Academy of Sciences of the United States of America. 2012. www.pnas.org/cgi/doi/10.1073/pnas.1201797109.

[16] Fitzpatrick AW, Knowles TP, Waudby CA, Vendruscolo M, Dobson CM. Inversion of the balance between hydrophobic and hydrogen bonding interactions in protein folding and aggregation. PLoS Computational Biology. 2011;7(10) e1002169.

[17] Rutledge SE, Volkman HM, Schepartz A. Molecular recognition of protein surfaces: high affinity ligands for the CBP KIX domain. Journal of the American Chemical Society. 2003;125(47) 14336-47.

[18] Pace CN, Fisher LM, Cupo JF. Globular protein stability: aspects of interest in protein turnover. Acta Biologica et Medica Germanica. 1981;40(10-11) 1385-92.

[19] Privalov PL, Gill SJ. Stability of protein structure and hydrophobic interaction. Advances in Protein Chemistry. 1988;39 191-234.

[20] Tsai AM, Udovic TJ, Neumann DA. The inverse relationship between protein dynamics and thermal stability. Biophysical Journal. 2001;81(4) 2339-43.

[21] Scandurra R, Consalvi V, Chiaraluce R, Politi L, Engel PC. Protein stability in extremophilic archaea. Frontiers in Bioscience: a journal and virtual library. 2000;5 D787-95.

[22] Hossain Z, Nouri MZ, Komatsu S. Plant cell organelle proteomics in response to abiotic stress. Journal of Proteome Research. 2012;11(1) 37-48.

[23] Gregersen N, Bross P, Vang S, Christensen JH. Protein misfolding and human disease. Annual Review of Genomics and Human Genetics. 2006;7 103-24.

[24] Amudha J, Balasubramani G. Recent moleular advances to combat abiotic stress tolerance in crop plants. Biotechnology and Molecular Biology Review. 2011;6(2) 31-58.

[25] Scharnagl C, Reif M, Friedrich J. Stability of proteins: temperature, pressure and the role of the solvent. Biochimica et Biophysica Acta. 2005;1749(2) 187-213.

[26] Hernandez G, Jenney FE, Jr., Adams MW, LeMaster DM. Millisecond time scale conformational flexibility in a hyperthermophile protein at ambient temperature. Proceedings of the National Academy of Sciences of the United States of America. 2000;97(7) 3166-70.

[27] Foord RL, Leatherbarrow RJ. Effect of osmolytes on the exchange rates of backbone amide protons in proteins. Biochemistry. 1998;37(9) 2969-78.

[28] Batchelor JD, Olteanu A, Tripathy A, Pielak GJ. Impact of protein denaturants and stabilizers on water structure. Journal of the American Chemical Society. 2004;126(7) 1958-61.

[29] Oro JRDX. Role of Co-Solute in Biomolecular Stability: Glucose, Urea and the Water Structure. Journal of Biological Physics. 2001;27 73-79. 
[30] Daniel RM, Dunn RV, Finney JL, Smith JC. The role of dynamics in enzyme activity. Annual Review of Biophysics and Biomolecular Structure. 2003;32 69-92.

[31] Kurkal V, Daniel RM, Finney JL, Tehei M, Dunn RV, Smith JC. Enzyme activity and flexibility at very low hydration. Biophysical Journal. 2005;89(2) 1282-7.

[32] Fields PA. Review: Protein function at thermal extremes: balancing stability and flexibility. Comparative Biochemistry and Physiology. Part A, Molecular \& Integrative Physiology. 2001;129(2-3) 417-31.

[33] Jaindl M, Popp M. Cyclitols protect glutamine synthetase and malate dehydrogenase against heat induced deactivation and thermal denaturation. Biochemical and Biophysical Research Communications. 2006;345(2) 761-5.

[34] Singh S, Singh A, Aziz MA, Waheed SM, Bhat R, Bhatnagar R. Thermal inactivation of protective antigen of Bacillus anthracis and its prevention by polyol osmolytes. Biochemical and Biophysical Research Communications. 2004;322(3) 1029-37.

[35] Simon LM, Kotorman M, Garab G, Laczko I. Effects of polyhydroxy compounds on the structure and activity of alpha-chymotrypsin. Biochemical and Biophysical Research Communications. 2002;293(1) 416-20.

[36] Carvalho AS, Santos AM, Neves-Petersen MT, Petersen SB, Aires-Barros MR, e Melo EP. Conformational states of HRPA1 induced by thermal unfolding: effect of low molecular weight solutes. Biopolymers. 2004;75(2) 173-86.

[37] Gekko K, Timasheff SN. Thermodynamic and kinetic examination of protein stabilization by glycerol. Biochemistry. 1981;20(16) 4677-86.

[38] Alliance Protein Laboratories Inc: Circular Dichroism: http://www.ap-lab.com/circular_dichroism.htm\#CD_secondary (accessed 26 July 2012).

[39] Ortbauer M, Popp M. Functional role of polyhydroxy compounds on protein structure and thermal stability studied by circular dichroism spectroscopy. Plant Physiology and Biochemistry : PPB / Societe francaise de physiologie vegetale. 2008;46(4) 428-34.

[40] Runzler D, Huber C, Moll D, Kohler G, Sara M. Biophysical characterization of the entire bacterial surface layer protein SbsB and its two distinct functional domains. The Journal of Biological Chemistry. 2004;279(7) 5207-15.

[41] Kaushik JK, Bhat R. Why is trehalose an exceptional protein stabilizer? An analysis of the thermal stability of proteins in the presence of the compatible osmolyte trehalose. The Journal of Biological Chemistry. 2003;278(29) 26458-65.

[42] Melo EP, Chen L, Cabral JM, Fojan P, Petersen SB, Otzen DE. Trehalose favors a cutinase compact intermediate off-folding pathway. Biochemistry. 2003;42(24) 7611-7.

[43] Neelon K, Schreier HJ, Meekins H, Robinson PM, Roberts MF. Compatible solute effects on thermostability of glutamine synthetase and aspartate transcarbamoylase from Methanococcus jannaschii. Biochimica et Biophysica Acta. 2005;1753(2) 164-73. 
[44] Myers JK, Pace CN, Scholtz JM. Denaturant m values and heat capacity changes: relation to changes in accessible surface areas of protein unfolding. Protein Science : A Publication of the Protein Society. 1995;4(10) 2138-48.

[45] Consalvi V, Chiaraluce R, Giangiacomo L, Scandurra R, Christova P, Karshikoff A, Knapp S, Ladenstein R. Thermal unfolding and conformational stability of the recombinant domain II of glutamate dehydrogenase from the hyperthermophile Thermotoga maritima. Protein Engineering. 2000;13(7) 501-7.

[46] Shastry MC, Eftink MR. Reversible thermal unfolding of ribonuclease T1 in reverse micelles. Biochemistry. 1996;35(13) 4094-101.

[47] Liu PF, Park C. Selective Stabilization of a Partially Unfolded Protein by a Metabolite. Journal of Molecular Biology. 2012. DOI 10.1016/j.jmb.2012.05.044.

[48] Brueschweiler R, Wright PE. NMR Order Parameters of Biomolecules: A New Analytical Representation and Application to the Gaussian Axial Fluctuation Model. Journal of the American Chemical Society. 1994;116(18) 8426-8427.

[49] Harder T, Borg M, Bottaro S, Boomsma W, Olsson S, Ferkinghoff-Borg J, Hamelryck T. An efficient null model for conformational fluctuations in proteins. Structure. 2012;20(6) 1028-39.

[50] Hansen DF, Vallurupalli P, Kay LE. Using relaxation dispersion NMR spectroscopy to determine structures of excited, invisible protein states. Journal of Biomolecular NMR. 2008;41(3) 113-20.

[51] Schanda P, Brutscher B, Konrat R, Tollinger M. Folding of the KIX domain: characterization of the equilibrium analog of a folding intermediate using 15N/13C relaxation dispersion and fast $1 \mathrm{H} / 2 \mathrm{H}$ amide exchange NMR spectroscopy. Journal of Molecular Biology. 2008;380(4) 726-41.

[52] Tollinger M, Skrynnikov NR, Mulder FA, Forman-Kay JD, Kay LE. Slow dynamics in folded and unfolded states of an SH3 domain. Journal of the American Chemical Society. 2001;123(46) 11341-52.

[53] Tollinger M, Kloiber K, Agoston B, Dorigoni C, Lichtenecker R, Schmid W, Konrat R. An isolated helix persists in a sparsely populated form of KIX under native conditions. Biochemistry. 2006;45(29) 8885-93.

[54] Horng JC, Tracz SM, Lumb KJ, Raleigh DP. Slow folding of a three-helix protein via a compact intermediate. Biochemistry. 2005;44(2) 627-34.

[55] Chen TH, Murata N. Enhancement of tolerance of abiotic stress by metabolic engineering of betaines and other compatible solutes. Current Opinion in Plant Biology. 2002;5(3) 250-7.

[56] Sheveleva E, Chmara W, Bohnert HJ, Jensen RG. Increased Salt and Drought Tolerance by D-Ononitol Production in Transgenic Nicotiana tabacum L. Plant Physiology 1997;115(3) 1211-1219. 
[57] Waditee R, Bhuiyan NH, Hirata E, Hibino T, Tanaka Y, Shikata M, Takabe T. Metabolic engineering for betaine accumulation in microbes and plants. The Journal of Biological Chemistry. 2007;282(47) 34185-93.

[58] Tarczynski MC, Jensen RG, Bohnert HJ. Expression of a bacterial mtlD gene in transgenic tobacco leads to production and accumulation of mannitol. Proceedings of the National Academy of Sciences of the United States of America. 1992;89(7) 2600-4.

[59] Sheveleva EV, Marquez S, Chmara W, Zegeer A, Jensen RG, Bohnert HJ. Sorbitol-6phosphate dehydrogenase expression in transgenic tobacco. High amounts of sorbitol lead to necrotic lesions. Plant Physiology. 1998;117(3) 831-9.

[60] Holmström K-O, Mäntylä E, Welin B, Mandal A, Palva ET, Londesborough OETJ. Drought tolerance in tobacco. Nature. 1996;379 683-684.

[61] Luo D, Niu X, Yu J, Yan J, Gou X, Lu BR, Liu Y. Rice choline monooxygenase (Os$\mathrm{CMO}$ ) protein functions in enhancing glycine betaine biosynthesis in transgenic tobacco but does not accumulate in rice (Oryza sativa L. ssp. japonica). Plant Cell Reports. 2012. DOI 10.1007/s00299-012-1276-2

[62] Singh AK, Kumar R, Pareek A, Sopory SK, Singla-Pareek SL. Overexpression of Rice CBS Domain Containing Protein Improves Salinity, Oxidative, and Heavy Metal Tolerance in Transgenic Tobacco. Molecular Biotechnology. 2012. DOI 10.1007/ s12033-011-9487-2

[63] Wu L, Zhou M, Shen C, Liang J, Lin J. Transgenic tobacco plants over expressing cold regulated protein CbCOR15b from Capsella bursa-pastoris exhibit enhanced cold tolerance. Journal of Plant Physiology. 2012.

[64] Hong Z, Lakkineni K, Zhang Z, Verma DP. Removal of feedback inhibition of delta(1)-pyrroline-5-carboxylate synthetase results in increased proline accumulation and protection of plants from osmotic stress. Plant Physiology. 2000;122(4) 1129-36.

[65] Pedone E, Saviano M, Rossi M, Bartolucci S. A single point mutation (Glu85Arg) increases the stability of the thioredoxin from Escherichia coli. Protein Engineering. 2001;14(4) 255-60.

[66] Vieille C, Zeikus GJ. Hyperthermophilic enzymes: sources, uses, and molecular mechanisms for thermostability. Microbiology and molecular biology reviews: MMBR. 2001;65(1) 1-43.

[67] van den Burg B, Eijsink VG. Selection of mutations for increased protein stability. Current Opinion in Biotechnology. 2002;13(4) 333-7. 
